Methods We conducted a systematic review on observational studies of metal levels from biological matrices, and dietary and occupational/environmental sources among PD patients and controls. We searched the PubMed/MEDLINE, EMBASE and Cochrane databases up to July 2020. Metal species included manganese, iron, copper, lead, mercury, aluminum, calcium, selenium, zinc, magnesium, cadmium, chromium and nickel, and the outcome was idiopathic PD. We applied an adapted Newcastle-Ottawa Scale (NOS) and a previously established exposure assessment rating to evaluate each individual study. We then performed meta-analyses with random-effects model.

Results 80 case-control studies were included, of which 69 were graded as low or moderate quality. The majority of casecontrol studies were hospital-based and applied biomonitoring approaches to quantify metal levels after disease diagnosis. Studies on copper, iron, manganese and zinc were most prevalent. Meta-analyses showed no significant PD risk for these metals and heterogeneity among studies was substantial. Furthermore, 5 cohort studies were retained, but the population source, metal exposure and follow-up period were heterogeneous.

Conclusion The level of evidence on metal exposure and PD risk is limited and no consensus can be drawn from the literature. Reverse causality cannot be ruled out by existing biomonitoring studies. Studies assessing metal levels before disease onset are needed to improve our understanding of the role of metals in the etiology of PD.

\section{P-279 RETURN TO WORK AFTER PARENTAL LEAVE: PERSPECTIVE OF BRAZILIAN WOMEN}

${ }^{1}$ Leticia Santos, Vivian Mininel. 'Universidade Federal de São Carlos, Brazil

\subsection{6/OEM-2021-EPI.259}

Introduction maternity leave is a constitutional right in Brazil, but the payment/benefits is restricted to women who contribute to the social security system. Although women represent half of the labor workforce around the world, they are still mostly responsible for family and child-rearing which compromises the process of return to work after birth.

Objective to understand the return to work after maternity leave from the perspectives of Brazilian working women.

Methods qualitative study with mothers that gave birth and return to work regardless of kind of job. Until now, four women have participated in online interviews following a validated script of open questions. Data analysis has been done simultaneously of data collected through thematic approach and the sample will be delimited by saturation. This research was approved by the Research Ethics Committee of the Federal University of Sao Carlos, Brazil (all ethical concerns have been respected).

Results return to work influenced the continuation of breastfeeding, including home office: 'I was in front of the computer and breastfeeding'. There is a duality when women talk about job and the care with the babies: 'the good thing is that I manage to stay with him all the time [...] and at the same time it also becomes exhausting to stay at home for a long time'. Women report that the work was invaded by the needs of their children causing dissatisfaction and frustration with performance after returning: 'I feel more encouraged to work [...] but my career is over, at least in the pandemic there is no way', especially during pandemic with all day care centers closed.

Conclusion the burden of responsibility in caring for children falls on women impacting performance at work and also breastfeeding.

\section{P-280 METALWORKING FLUIDS AND CANCER INCIDENCE IN THE UAW-GM AUTOWORKERS COHORT}

${ }^{1}$ Hilary Colbeth, Kevin Chen, Sally Picciotto, Sadie Costello, Ellen Eisen. ${ }^{1}$ University of California Berkeley, United States

\subsection{6/OEM-2021-EPI.260}

Introduction Metalworking fluids (MWF) are complex mixtures of oils and chemical additives used to cool and lubricate metal machining operations. Previous studies have reported increased risk of specific cancers associated with MWF exposure.

Objectives This report broadly examines cancer incidence in the United Auto Workers-General Motors (UAW-GM) cohort exposed to MWFs with extended follow-up (through 2015). The outcomes of interest were melanoma, leukemia, nonHodgkin lymphoma and cancers of the colon, rectum, pancreas, esophagus, stomach, larynx, lung and bronchus, breast, prostate, kidney and renal pelvis, and bladder.

Methods The cohort includes 39,132 workers followed for cancer incidence in Detroit area Surveillance, Epidemiology, and End Results Program (SEER) and Michigan cancer registries, from 1973-2015. Cox models yielded estimates of adjusted hazard ratios (HR) with categorical variables for lagged cumulative exposure to each MWF (straight, soluble, and synthetic).

Results There were 7,809 cancer cases of interest. Over 43 years of follow-up, the incidence of several types of cancers was significantly elevated in relation to at least one type of MWF; exposure-response patterns were consistent with prior reports from this cohort. We found significantly increased incidence of stomach and kidney cancer associated with higher levels of straight fluid exposure and increased rectal and pancreatic cancer with increasing synthetic fluid exposure. Only Non-Hodgkin lymphoma was associated with soluble MWF, with HRs significantly elevated in the highest exposure category at 1.70 (95\% Confidence Interval (CI): 1.13-2.54).

Conclusions Our results provide further evidence of associations between MWF exposure and several types of cancer. This study summarizes information on the incidence of the fourteen cancer types with reduced bias from both the healthy worker hire effect and left truncation. However, the HRs presented do not address potential downward bias from the healthy worker survivor effect which may be necessary to correct in future targeted analyses.

\section{P-281 IDENTIFYING RETURN-TO-WORK TRAJECTORIES AMONG BREAST CANCER SURVIVORS USING SEQUENCE ANALYSIS}

${ }^{1}$ Mélanie Bertin, Yves Roquelaure, Elise Rubion, Bertrand Porro, Fabien Gilbert. 'École des hautes études en santé publique (EHESP), France

10.1136/OEM-2021-EPI.261 
Introduction The return-to-work (RTW) process after breast cancer (BC) can be complex. Simple static measures used to assess RTW may ignore this dynamic multi-stage process that are yet important for targeting interventions aimed at reducing poor RTW outcomes.

Objectives Our aim was to identify RTW trajectories after BC using the sequence analysis method and describe their underpinning personal, medical, psychological and occupational factors.

Methods This study is based on the ELCCA II cohort (Angers, France) that recruited 128 women diagnosed with BC between February 2015 and April 2016. Among them, 96 were still participating at the four-year follow-up. An occupational calendar was used to assess their situation every 6 months from their diagnostic up to 4 years later. Women that did not complete their occupational calendar, that were inactive (retired, disability pension) at the time of their diagnosisic were excluded leading to a final sample of 52 breast cancer survivors (BCS). Sequence analysis was used to identify RTW trajectories among BCS. Patterns of RTW trajectories were described by socio-demographic, medical, occupational and the functional scales of the EORTC-QLQ-C30.

Results Three types of RTW trajectories were identified among BCS by sequence analysis: long sickness absence with progressive RTW $(n=18)$, short sickness absence and full time RTW ( $\leq 6$ months) $(n=27)$, short sickness absence with part-time RTW $(n=7)$. Socio-demographic, medical and trend in functional scales during the first year after diagnosis were shown to vary according RTW trajectories.

Conclusion The application of sequence analysis highlights the dynamic process of RTW among BCS. It captures trajectories of multiple states and transitions that provided a holistic and diachronic approach of RTW. Three different patterns of RTW trajectories after breast cancer were identified. However, these results have to be confirmed by using a larger sample of BCS.

\section{P-286 AN INVENTORY OF OCCUPATIONAL CANCER STUDIES AND THEIR EXPOSURE ASSESSMENT METHODS IN IRAN}

'Bayan Hosseini, Amy Hall, Kazem Zendehdel, Hans Kromhout, Felix Onyije, Rahmatollah Moradzadeh, Maryam Zamanian, Joachim Schuz, Ann Olsson. 'International Agency for Research on Cancer, France

\subsection{6/OEM-2021-EPI.262}

Introduction The presence of occupational carcinogens in workplaces in Iran are not well characterized, and little is known about the burden of occupational cancer in Iran and other Middle East countries.

Objectives This study aimed to provide a comprehensive overview of occupational cancer studies in Iran and describe how exposures were assessed and assigned in those studies.

Methods Studies addressing exposure to and health effects from carcinogens in Iran until the end of 2020 were identified through three databases (PubMed, Web of Science, and Google Scholar). Two reviewers independently screened the articles according to the PRISMA (Preferred Reporting Items for Systematic Reviews and Meta-Analyses) guideline. Data extracted from each eligible paper included geographical region, study design, occupation, industry, exposure agents, population size/sample size, exposure assessment methods, and cancer sites.
Results Forty-nine publications from inception to 2020 (1 cohort, 11 case-control, 34 exposure monitoring studies, and 3 other) were included. The case-control studies used selfreported job titles and/or self-reported exposures to estimate the associated risk of cancer, while exposure monitoring studies applied exposure measurements (including personal and stationary air sampling).

Conclusion Occupational cancer epidemiology in Iran is at an early stage, as evidenced by relatively small number of published articles on this topic. A coherent strategy to assess occupational exposures and cancer burden in Iran should include systematic inventory and monitoring of exposure to carcinogens for use in hazard control and research, as well as targeted tools and studies to assess unique occupational environments and their potential associated cancer hazards.

\section{P-290 NUMBER OF JOBS, JOBS DURATION AND RISK OF PROSTATE CANCER: A CASE-CONTROL STUDY IN MONTREAL, CANADA}

${ }^{1}$ Charlotte Salmon, Marie-Elise Parent. 'Institut national de la recherche scientifique, Université du Québec, Canada

\subsection{6/OEM-2021-EPI.263}

Introduction Successive job changes may reflect precarious employment, job dissatisfaction or promotions, all of which can result in accrued stress, disrupted social connections and health-altering circumstances and behaviours. Evidence is lacking on the potential role of job stability in cancer development.

Objectives We investigated the association between the number of jobs held and jobs duration over the career, and the risk of prostate cancer, overall and by cancer aggressiveness.

Methods Data from the Prostate Cancer \& Environment Study, a population-based case-control study conducted in 2005-2012 in Montreal, Canada were used. Incident cases $(n=1,931)$, aged $<76$ years, were ascertained across hospitals in the Montreal area. Population controls $(n=1,994)$ from the same area, frequency-matched to cases by age ( \pm 5 years), were identified from the electoral list. Information on the number of paid jobs held for $\geq 1$ year throughout the career and the duration of each job was elicited during face-to-face interviews. Unconditional logistic regression was used to assess odds ratios (OR) and 95\% confidence intervals (CI), adjusting for age, ancestry and education.

Results Subjects had held 5 jobs, on average ( \pm 3 ), with a mean job duration of 11.1 years $( \pm 7.9)$. A greater number of jobs held was associated with a higher risk of prostate cancer. The OR for men who had had $>6$ jobs was $1.23(95 \%$ CI $=1.04-1.46$ ), compared to men who had held $1-3$ jobs, with no differences according to cancer aggressiveness. Conversely, men who had held shorter jobs, on average, had a higher risk of prostate cancer, following a dose-response pattern (p-trend 0.047). The association was more pronounced for non-aggressive tumours.

Conclusion Our results suggest that job stability throughout the career may have a protective effect against prostate cancer. Further in-depth analyses are warranted. These findings are novel and require replication. 